\title{
Long non-coding RNAs drive metastatic progression in melanoma (Review)
}

\author{
POURIA AKHBARI ${ }^{1}$, ADRIAN WHITEHOUSE $^{2,3}$ and JAMES R. BOYNE ${ }^{1}$ \\ ${ }^{1}$ Centre for Skin Sciences, University of Bradford, Bradford; ${ }^{2}$ School of Molecular and Cellular Biology, \\ Faculty of Biological Sciences and ${ }^{3}$ Astbury Centre for Structural Molecular Biology, University of Leeds, Leeds, UK
}

Received July 7, 2014; Accepted August 21, 2014

DOI: 10.3892/ijo.2014.2691

\begin{abstract}
Metastatic melanoma is the leading cause of skin-cancer related deaths and while in recent years some progress has been made with targeted therapies, there remains an urgent unmet need for novel therapeutic treatments and reliable diagnostic, prognostic and predictive biomarkers. The emergence of next generation sequencing (NGS) has seen a growing appreciation for the role played by non-coding genomic transcripts in regulating gene expression and by extension impacting on disease progression. The long non-coding RNAs (lncRNAs) represent the most enigmatic of these new regulatory molecules. Our understanding of how lncRNAs regulate biological functions and their importance to disease aetiology, while still limited, is rapidly improving, in particular with regards to their role in cancer. Herein we review the identification of several lncRNAs shown to impact on melanoma disease progression and discuss how these molecules are operating at the molecular level.
\end{abstract}

\section{Contents}

1. Introduction

2. Long non-coding RNAs drive metastatic progression in melanoma

3. Conclusions

\section{Introduction}

Malignant melanoma (MM) has one of the fastest-rising incidences of all cancers (1). This, coupled with the poor prognosis for patients in stages III and IV of the disease, is causing a growing healthcare burden on society. At the molecular level, rather than being a single disease, melanoma comprises

Correspondence to: Dr James R. Boyne, Centre for Skin Sciences, University of Bradford, Richmond Rd, Bradford BD7 1DP, UK E-mail: j.boyne@bradford.ac.uk

Key words: melanoma, long non-coding RNA, metastasis a heterogeneous group of disorders that harbour distinct aberrations in diverse cellular processes such as cell cycle regulation, cell signalling, cell adhesion, cell differentiation and apoptosis (2). Such heterogeneity suggests that multiple mechanisms are involved in disease aetiology and this is reflected in the contribution of both different mutations and differential gene and protein expression associated with MM development and progression $(3,4)$.

Our understanding of genomics is currently undergoing a paradigm shift. The past decade has seen staggering advances in next generation sequencing (NGS), precipitating an explosion of genome-wide transcriptome studies, culminating in 2012 with the completion of the ENCODE project (5). Large scale cDNA sequencing and interrogation of whole chromosome tiling arrays across a variety of cell types reveal that $>90 \%$ of genomic nucleotides are transcribed (6). Perhaps the most surprising discovery to emerge from these studies is that only a tiny percentage of the RNA transcripts synthesised are fated to code for protein, with the vast majority being comprised of non-coding RNA. This realisation eschews the central dogma of molecular biology (7) and has ushered in a new era of non-coding RNA research that is focused on unpicking the functional significance of these prevalent transcripts $(8,9)$.

Non-coding RNAs can be broadly defined according to their size as either short (<200 nt) or long (>200 nt). Short non-coding RNAs are well-characterised, particularly those involved in the production and operation of gene expression machinery such as ribosomal RNA, transfer RNA, small-nuclear RNA and small-nucleolar RNA. More recently, short non-coding RNAs that function to regulate, rather than operate gene expression have been intensely investigated and can be further divided into three main subcategories: PIWI-associated RNAs, which regulate transposable elements, small interfering RNAs and microRNAs (miRNAs), which mediate post-transcriptional silencing of mRNA and the recently described transcription initiation RNAs, which appear to instigate gene expression at the promoter level. Of these, miRNAs are the best understood and function to regulate both fundamental processes in biology, such as proliferation, apoptosis and differentiation, while also contributing significantly to disease aetiology, most notably cancer (10), but also cardiovascular disease (11), stroke (12) and several neurological disorders $(13,14)$. The functional role played by miRNAs in the establishment and metastatic progression of melanoma was recently reviewed (15) and 
compiling data from several groups has enabled the coupling of differential miRNA expression in MM to specific target protein modulation and critically downstream functional consequence for disease (16-24). Such studies represent an emerging area of research, indeed, the prevalence of miRNAs in the human transcriptome continues to expand as deep-sequencing technologies improve, with current estimates identifying $\sim 9,000$ small non-coding RNAs, $\sim 1,100$ of which are potentially functional miRNA transcripts (5). However, this number is dwarfed by the pervasiveness of the long non-coding RNA (lncRNA) transcripts, with GENCODE identifying a staggering 22,444 non-coding transcripts $>200 \mathrm{nt}$ in length and more recent studies estimating this number to be nearer $32,000(25)$.

In contrast to miRNA, our understanding of lncRNA function is in its infancy, however, huge efforts are being made to fill this knowledge gap, particularly in relation to their role in disease. The importance of such study is underlined by what we do understand about a handful of lncRNAs involved in fundamental aspects of biology. Most notable of these are the lncRNAs XIST, which inactivates gene expression from the $\mathrm{X}$-chromosome during dosage equalisation and HOTAIR, a lncRNA that acts in trans via the Polycomb repressor complex to regulate genes at a distance, intimating that lncRNA are capable of more complex modes of gene regulation $(26,27)$. In parallel to their role in the regulation of cellular processes and akin to their smaller cousins, the miRNAs, it is the involvement of IncRNAs in the development and progression of cancer that drives much of the current research on these transcripts. This review will focus on recent advances made in the discovery of IncRNAs with a functional role in the progression of melanoma metastasis.

\section{Long non-coding RNAs drive metastatic progression in melanoma}

The prognosis for patients with early stage, localised melanoma is favourable, with 10 -year survival rates approaching $90 \%$, depending on Breslow thickness, ulceration and mitotic index (28). This prognosis deteriorates rapidly with the onset of regional metastasis (10-year survival of around 50\%) and for patients with stage IV melanoma and distant metastasis, 5-year survival rates are $<10 \%$ (29). Clearly, metastatic progression in melanoma severely impacts on patient health and there is an urgent need to expand our understanding of the mechanisms underpinning this process. Numerous studies investigating the functional role played by lncRNA in cancer suggest that they are functioning to regulate events intimately associated with the metastatic transition, such as cell migration and tissue invasion $(30,31)$. Here we will review our current understanding of lncRNAs known to impact on melanoma and consider what we understand about the functional mechanisms involved.

HOTAIR. HOTAIR is a member of an exclusive, but growing, family of lncRNAs that have comparatively well-defined cellular functions, in this case the epigenetic regulation of gene expression. HOTAIR is transcribed from the HOXC cluster and interacts with the Polycomb repressive complex 2 (PRC2), which it then trafficks to the HOXD cluster, facilitating transcriptional silencing of this $40 \mathrm{~kb}$ region (27). In addition to regulating the expression of this specific genomic region, HOTAIR also appears to regulate the gene expression at hundreds of other genomic locations via an interaction with the LSD1/CoREST/REST complex, which enables recruitment of PRC2 and LSD1 to chromatin for coupled histone H3K27 methylation and K4 demethylation (32). With such a pivotal role in epigenetic modification and transcriptional activity, it is not surprising that HOTAIR expression is deregulated in numerous cancers, including breast, lung, colorectal,pancreatic, ovarian hepatocellular carcinoma and gastrointestinal stromal tumours (GIST) (33-42), indeed HOTAIR has been shown to reprogram the cancer epigenome in breast cancer towards a metastatic state, cementing its position as a molecule of huge importance in cancer biology (43).

In order to determine if HOTAIR and other lncRNAs known to be deregulated across a range of cancers are upregulated in metastatic melanoma, Tang et al carried out quantitative PCR (qPCR)-based expression profiling on matched primary melanoma versus lymph node tumour samples from three patients (44). These analyses revealed two things: i) HOTAIR expression is dramatically upregulated by $\sim 100$-fold in metastatic versus primary melanoma; and ii) several other lncRNAs (HULC, MALAT-1, MEG3, NEAT1 and UCA1), which are associated with metastatic progression across a range of cancers were not differently expressed in metastatic melanoma, suggesting that lncRNAs contribute to metastasis in a cancer-specific manner (44). Moreover, it appears that elevated HOTAIR expression is a determining factor for the metastatic state, at least in vitro, with siRNA-mediated depletion of HOTAIR in the metastatic melanoma cell line, A375, resulting in significant decreases in both cell motility and invasion. These properties underpin metastasis, which involves the destruction of the basement membrane and migration of the tumour cells into the connective tissues before spreading to the lymph nodes and distal sites. The basement membrane is largely comprised of type IV collagen, which is degraded by matrix metalloproteinases (MMPs), specifically MMP-2 and MMP-9 (45). Intriguingly, in situ zymography assays carried out by Tang et al (44) show that depletion of HOTAIR in A375 cells resulted in reduced activity of MMP-2 and MMP-9, suggesting a possible mechanism for HOTAIR-mediated metastasis (Fig. 1A), a theory supported by recent data indicating that MMP gene expression is heavily regulated at the epigenetic level (46). Considering HOTAIR's association with epigenetic reprogramming and given the potential use of epigenetic drugs that target MMPs to treat melanoma (47), there is an urgent need to develop a keener understanding of such mechanisms.

SPRY4-IT1. HOTAIR appears destined to be involved in the aetiology of the majority of cancers, melanoma included. However, it was not the first lncRNA to be associated with melanoma, this accolade goes to the SPRY4-IT1 transcript, a lncRNA that is derived from an intron of the SPRY4 gene (48). In contrast to HOTAIR, very little is known about the cellular function of SPRY4-IT1, which was reported to be differentially expressed in melanoma by Khaitan et al (48). The authors utilised a microarray approach to determine differences in lncRNA expression between the stage III melanoma cell line, WM1552C, and control melanocytes. Alongside this, patient 
A

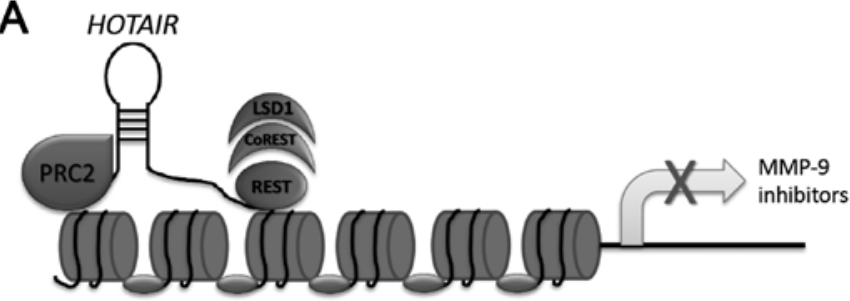

B

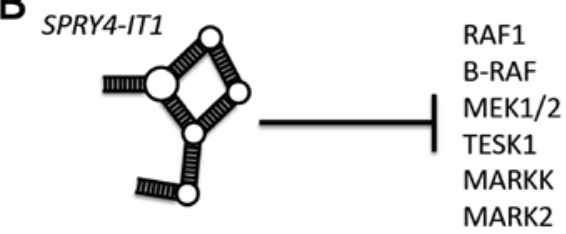

C

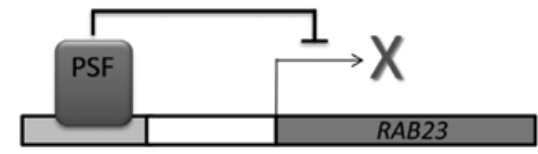

LIme23

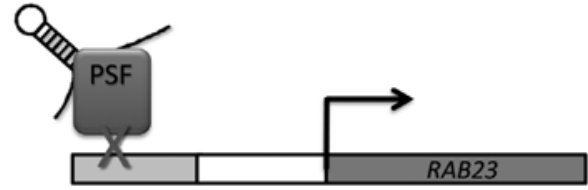

D

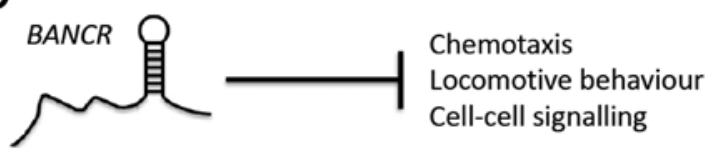

Figure 1. Putative functional mechanisms for melanoma-associated long non-coding RNAs (lncRNAs). (A) HOTAIR is likely to be downregulating MMP-9 expression by functioning in trans to recruit chromatin modification complexes to upstream regulatory regions. (B) The cellular mechanisms underpinning SPRY4-ITI function remain to be elucidated; however, it is likely that MAPK pathways will be targeted given the regulatory role of SPRY4 in these signalling networks. (C) Llme23 physically interacts with protein-associated splicing factor (PSF) leading to its disassociation from upstream regulatory elements in the RAB23 locus, thereby leading to overexpression of $R A B 23$ and oncogenesis. (D) Depletion of $B R A F$-activated non-coding RNA $(B A N C R)$ resulted in the differential expression of 88 genes that were enriched for transcripts with gene ontology terms associated with metastasis.

samples isolated from stage I-IV disease were also assessed and results aligned with cell line data to identify lncRNA transcripts that were differentially expressed in both experimental systems. This careful approach, validated by NGS, identified four lncRNA transcripts, three of which were disregarded due to their location in the $3^{\prime}$ UTR of protein-coding genes and concerns over the technical difficulties this would present for downstream analysis, the remaining transcript, which also displayed the greatest differential expression ( $\sim 2$-fold), was SPRY4-ITI (48).

In addition to increased expression in melanoma, the SPRY4-ITI lncRNA is also of interest due to its predicted secondary structure. Analysis of SPRY4-IT1 using RNAfold and RNAstructure $(49,50)$ revealed several putative regulatory motifs, including three nested helices and two 'pyknons' motifs (48). The presence of pyknons (non-random, genome-wide motifs) is particularly intriguing as it has been suggested that these elements play a role in post-transcriptional gene silencing (51), indicating that SPRY4-IT1 may directly affect gene expression, something yet to be confirmed experimentally. Depletion of SPRY4-IT1 in A375 cells using siRNA and scramble controls resulted in decreased metabolic viability and increased apoptosis via MTT and Annexin V assay, respectively. Moreover, SPRY4-IT1-depleted A375 cells exhibited significant reduction in both cell invasion and cell motility when compared with scramble siRNA-transfected control. Importantly, each of these phenotypic changes can be ascribed to reduced SPRY4-IT1, as knockdown of the lncRNA did not alter the expression of its 'host' gene SPRY4. Furthermore, overexpression of SPRY4-IT1 in LOX-IMV1, a metastatic melanoma cell line shown to express only low-levels of SPRY4-ITI, significantly increased cell motility in a wound healing assay (48).

A defined cellular function for SPRY4-ITI remains elusive, however, it has recently been linked with the aetiology of preeclampsia and oesophageal squamous cell carcinoma $(52,53)$. One possible clue may reside in the geographical location of SPRY4-IT1 within the intronic sequence of the SRYY4 gene, a property that may indicate a biological function that is linked to that of the 'host' gene (54). SPRY4 is a member of the Sprouty family of Ras/ERK inhibitors proteins that prevent the formation of active GTP-RAS and are therefore considered to be putative tumour suppressors $(55,56)$. Clearly, SPRY4-IT1 is not operating as a tumour suppressor in melanoma; however, it may manipulate similar intrinsic cellular pathways, such as Ras/ERK (Fig. 1B). Future experiments, such as investigating the effect of Ras/ERK inhibitors on increased cellular motility following SPRY4-ITI overexpression will likely prove informative and are of clinical importance given emerging targeted therapies for melanoma that focus on MAPK-signalling (57).

Llme23. Thus far, we have considered lncRNAs identified due to their differential expression in metastatic melanoma. The lncRNA Llme23 differs in this respect as while it appears to be exclusively expressed in melanoma, it was first described using assays designed to identify functional lncRNA partners to the polypyrimidine tract-binding protein-associated splicing factor (PSF) (58). The PSF protein is a somewhat intriguing molecule that was originally thought to operate as a splicing factor, having been identified in spliceosomal extracts (59). However, subsequent analysis has revealed a tumour-suppressor function for this protein. Study carried out in mouse models demonstrate that PSF is able to regulate the transcriptional activity in multiple proto-oncogenes via its DNA-binding domain (DBD), which interacts with the regulatory regions of these targets, repressing their expression (60).

Fascinatingly, this tumour-suppressor function is eradicated by the binding of a mouse retrotransposon IncRNA, VL30-1, to the RNA-binding domain motifs present in PSF. Moreover, retroviral transmission of VL30-1 to human melanoma cells promoted metastatic progression in immunocompromised mice, suggesting a possible role for PSF in the aetiology of metastatic melanoma $(61,62)$. While attention-grabbing, the absence of a human homologue of the mouse VL30-1 lncRNA raised doubts regarding the existence of a similar intrinsic mechanism in humans. However, recent study by 
Wu et al describes a human IncRNA that interacts directly with PSF in order to drive melanoma tumour formation (58). Here an RNA-SELEX approach was utilised to enrich for human RNAs that bind PSF and via subsequent cDNA library construction and electromobility gel-shift assays, a 1,600 nt lncRNA, termed Llme23, was identified that binds directly to PSF. These in vitro studies were also confirmed in vivo by RNA-immunoprecipitations and downstream functional experiments confirmed that Llme 23 binding to PSF inactivated PSF-mediated repression of $R A B 23$, confirming that the human Llme23 lncRNA also inhibits PSF's tumour-suppressor function (Fig. 1C), as is the case with the murine lncRNA VL30-1 and PSF in the mouse model system (61).

How then is Llme23 impacting on melanoma aetiology? Data investigating the effects of Llme 23 overexpression and depletion is more limited than for HOTAIR and SPRY4-ITI, however, Llme23-depleted YUSAC cells displayed a significant decrease in their ability to form colonies in soft-agar and most significantly, these same cells displayed $\sim 75 \%$ decrease in tumour volume at day 38 post-injection into nude mice (58). Taking into consideration the emerging literature around PSF and cancer $(63,64)$, it will be extremely interesting to observe the delineation of Llme23 function in the literature, which to date is restricted to the article discussed here.

BANCR. As discussed above, derailment of the Ras/ERK MAPK signalling cascade is extremely common in metastatic melanoma. Our understanding of the molecular events that underpin such deregulation took a conceptual leap forward in 2002 with the discovery of mutations in the v-RAF murine oncogene homologue $\mathrm{B}$ (BRAF) proto-oncogene (65). The significance of this finding is linked to the prevalence of BRAF mutations in cutaneous melanoma, with $>50 \%$ of tumours harbouring mutations in BRAF and crucially $>90 \%$ of these cases possessing the same BRAF ${ }^{\mathrm{V} 600 \mathrm{E}}$ substitution $(66,67)$. This realisation resulted in a focused effort to develop $\mathrm{BRAF}^{\mathrm{V} 600 \mathrm{E}}$-specific inhibitors and has resulted in the development and release of two drugs, vemurafenib and dabrafenib, which received approval by the FDA for the treatment of BRAF ${ }^{\mathrm{V} 600 \mathrm{E}}$ mutant melanoma in 2011 and 2013, respectively. Unfortunately, despite excellent response rates of $\sim 50 \%$, the vast majority of patients treated with these drugs relapse and progress to chemoresistant disease that is generally fatal. A number of mechanistic explanations have been identified to explain this relapse, with the majority linked to the reactivation of aberrant MARK-signalling, although other pathways (insulin growth factor receptor and platelet-derived growth factor receptor) have also been implicated (68). Clearly, there is an urgent need to better understand how oncogenic BRAF interacts with the cellular machinery to impact disease.

Flockhart et al set out with just this goal in mind as outlined in their recent article, which describes the use of RNA-seq on primary human melanocytes transduced with lentivirus expressing either $\mathrm{BRAF}^{\mathrm{V} 600 \mathrm{E}}$ or red fluorescent protein control in order to specifically identify differentially expressed lncRNAs (69). This approach represents an important shift away from other studies, which have generally focused on IncRNAs that are differentially expressed in cancer, without first addressing the cause of the observed differential expression and whether it is driven by oncogenic events or an artefact of tumour heterogeneity and genomic instability.

In addition to determining global transcriptomic changes in primary melanocytes expressing $\mathrm{BRAF}^{\mathrm{V} 600 \mathrm{E}}$, RNA-seq data were granted more clinical credence by concurrently analysing $\mathrm{BRAF}^{\mathrm{V} 600 \mathrm{E}}$-positive melanoma tissue samples. Indeed, results were processed through a rigorous study-flow that utilised cross-referencing with a publically available melanoma RNA-seq data set (70) and interrogation of the ENCODE RNA-seq data in order to validate that identified transcripts are actively transcribed in melanocytes. Finally, transcripts of interest were analysed using a previously described coding potential calculator (CPC) algorithm that discriminates coding from non-coding transcripts (71). This combinatorial approach identified a novel lncRNA that is overexpressed in $\mathrm{BRAF}^{\mathrm{V} 600 \mathrm{E}}$-positive melanocytes and melanoma, which the authors termed $B R A F$-activated non-coding RNA (BANCR) (69).

Functional experiments depleting BANCR in melanoma cells did not result in decreased viability and proliferation. However, consistent with HOTAIR, SPRY4-ITI and Llme23, depletion of $B A N C R$ did significantly reduce melanoma cell motility. Gene expression profiling carried out on Colo829 $\mathrm{BRAF}^{\mathrm{V} 600 \mathrm{E}}$-depleted melanoma cells using a cDNA microarray revealed that 88 genes were differentially expressed compared with control and significantly genes involved in cell motility were overrepresented among this number (Fig. 1D) (69). In order to gain some functional insight into how $B A N C R$ might be regulating cell migration, targets that displayed altered gene expression in BANCR-depleted cells and are associated with cell motility were investigated further. Interestingly, the chemokine CXCL11, the expression of which appears to be positively regulated by $B A N C R$, was able to rescue the reduced cell motility phenotype observed in BANCR-depleted melanoma cells. These data suggest a scenario where the $\mathrm{BRAF}^{\mathrm{V} 600 \mathrm{E}}$ mutation induces overexpression of $B A N C R$, which in turn then positively regulates expression of CXCL11 in order to promote cell migration. This observation may be of clinical importance as activation of the chemokine receptor, CXCR3, has been linked to lymph node metastasis in melanoma (72).

\section{Conclusions}

Over the past decade, the explosion of research on non-coding transcripts has left us in little doubt that our genomes exhibit great transcriptional complexity. However, our understanding of how this complexity links to function, if at all, remains far less assured. Clearly, lncRNAs are key players in cancer progression and exhibit huge potential as biomarkers and novel therapeutic targets for treatment. Perhaps the biggest challenge facing researchers is determining which of the many thousands of transcripts are truly functional. Classical single 'gene' experimental analysis remains central to this process, but with improved read-length technologies for NGS on the horizon it is likely that such studies will benefit greatly from improved functional annotation. A second challenge relates to our understanding of how IncRNA structure can be analysed and used to predict function and accurately identify target genes via bioinformatics. Certainly there is a long and 
somewhat uncharted road ahead. Flockhart et al (69), identified an additional 38 annotated lncRNA transcripts, in addition to $B A N C R$, that are regulated by $\mathrm{BRAF}^{\mathrm{V} 600 \mathrm{E}}$ and expressed in melanoma and similar reservoirs likely remain untapped from other melanoma RNA-seq data sets. Furthermore, the array of regulatory mechanisms that impact on non-coding RNA function continues to grow. Methyl-6-adenosine modification of RNA transcripts was recently shown to be a reversible event that is thought to regulate mRNA and lncRNA stability and has ushered yet another area of gene expression research, RNA epigenetics (73). While the challenges are numerous, the rewards are significant, characterisation of functional lncRNAs and their modes of action will provide exciting opportunities to augment and improve melanoma diagnosis, prognostic monitoring and targeted therapies.

\section{References}

1. Weyers W: The 'epidemic' of melanoma between under- and overdiagnosis. J Cutan Pathol 39: 9-16, 2012.

2. Lomas J, Martin-Duque P, Pons M and Quintanilla M: The genetics of malignant melanoma. Front Biosci 13 5071-5093, 2008.

3. Hill VK, Gartner JJ, Samuels Y and Goldstein AM: The genetics of melanoma: recent advances. Annu Rev Genomics Hum Genet 14: 257-279, 2013.

4. Bougnoux AC and Solassol J: The contribution of proteomics to the identification of biomarkers for cutaneous malignant melanoma. Clin Biochem 46: 518-523, 2013.

5. ENCODE Project Consortium: An integrated encyclopedia of DNA elements in the human genome. Nature 489: 57-74, 2012.

6. Clark MB, Amaral PP, Schlesinger FJ, et al: The reality of pervasive transcription. PLoS Biol 9: e1000625; discussion e1001102, 2011.

7. Crick F: Central dogma of molecular biology. Nature 227: $561-563,1970$

8. Graur D, Zheng Y, Price N, Azevedo RB, Zufall RA and Elhaik E: On the immortality of television sets: 'function' in the human genome according to the evolution-free gospel of ENCODE. Genome Biol Evol 5: 578-590, 2013.

9. Doolittle WF: Is junk DNA bunk? A critique of ENCODE. Proc Natl Acad Sci USA 110: 5294-5300, 2013.

10. Tessitore A,Cicciarelli G, Del Vecchio F, et al: microRNAs in the DNA damage/repair network and cancer. Int J Genomics 2014 820248, 2014.

11. Condorelli G, Latronico MV and Cavarretta E: microRNAs in cardiovascular diseases: current knowledge and the road ahead. J Am Coll Cardiol 63: 2177-2187, 2014

12. Yin KJ, Hamblin $M$ and Chen YE: Non-coding RNAs in cerebral endothelial pathophysiology: emerging roles in stroke. Neurochem Int: April 3, 2014 (Epub ahead of print).

13. Lukiw WJ and Alexandrov PN: Regulation of complement factor $\mathrm{H}(\mathrm{CFH})$ by multiple miRNAs in Alzheimer's disease (AD) brain. Mol Neurobiol 46: 11-19, 2012.

14. Filatova EV, Alieva AKh, Shadrina MI and Slominsky PA: MicroRNAs: possible role in pathogenesis of Parkinson's disease. Biochemistry (Mosc) 77: 813-819, 2012.

15. Völler D, Ott C and Bosserhoff A: MicroRNAs in malignant melanoma. Clin Biochem 46: 909-917, 2013.

16. Dar AA, Majid S, de Semir D, Nosrati M, Bezrookove V and Kashani-Sabet M: miRNA-205 suppresses melanoma cell proliferation and induces senescence via regulation of E2F1 protein. J Biol Chem 286: 16606-16614, 2011.

17. Braig S, Mueller DW, Rothhammer T and Bosserhoff AK: MicroRNA miR-196a is a central regulator of HOX-B7 and BMP4 expression in malignant melanoma. Cell Mol Life Sci 67: 3535-3548, 2010.

18. Mueller DW and Bosserhoff AK: MicroRNA miR-196a controls melanoma-associated genes by regulating HOX-C8 expression. Int J Cancer 129: 1064-1074, 2011.

19. Levati L, Pagani E, Romani S, et al: MicroRNA-155 targets the SKI gene in human melanoma cell lines. Pigment Cell Melanoma Res 24: 538-550, 2011.
20. Deng Y, Deng H, Bi F, et al: MicroRNA-137 targets carboxylterminal binding protein 1 in melanoma cell lines. Int J Biol Sci 7: 133-137, 2011.

21. Bemis LT, Chen R, Amato CM, et al: MicroRNA-137 targets microphthalmia-associated transcription factor in melanoma cell lines. Cancer Res 68: 1362-1368, 2008.

22. Segura MF, Hanniford D, Menendez S, et al: Aberrant miR-182 expression promotes melanoma metastasis by repressing $\mathrm{FOXO} 3$ and microphthalmia-associated transcription factor. Proc Natl Acad Sci USA 106: 1814-1819, 2009

23. Haflidadóttir BS, Bergsteinsdóttir K, Praetorius C and Steingrímsson E: miR-148 regulates Mitf in melanoma cells. PLoS One 5: e11574, 2010.

24. Karreth FA, Tay Y, Perna D, et al: In vivo identification of tumor-suppressive PTENceRNAs in an oncogenic BRAF-induced mouse model of melanoma. Cell 147: 382-395, 2011.

25. Volders PJ, Helsens K, Wang X, et al: LNCipedia: a database for annotated human IncRNA transcript sequences and structures. Nucleic Acids Res 41: D246-D251, 2013.

26. Brockdorff N, Ashworth A, Kay GF, et al: The product of the mouse $\mathrm{Xist}$ gene is a $15 \mathrm{~kb}$ inactive $\mathrm{X}$-specific transcript containing no conserved ORF and located in the nucleus. Cell 71: 515-526, 1992.

27. Rinn JL, Kertesz M, Wang JK, et al: Functional demarcation of active and silent chromatin domains in human HOX loci by noncoding RNAs. Cell 129: 1311-1323, 2007.

28. Balch CM, Gershenwald JE, Soong SJ, et al: Final version of 2009 AJCC melanoma staging and classification. J Clin Oncol 27: 6199-6206, 2009

29. Dickson PV and Gershenwald JE: Staging and prognosis of cutaneous melanoma. Surg Oncol Clin N Am 20: 1-17, 2011.

30. Zhang H, Chen Z, Wang X, Huang Z, He Z and Chen Y: Long non-coding RNA: a new player in cancer. J Hematol Oncol 6: 37, 2013.

31. Cheetham SW, Gruhl F, Mattick JS and Dinger ME: Long noncoding RNAs and the genetics of cancer. Br J Cancer 108: 2419-2425, 2013.

32. Tsai MC, Manor O, Wan Y, et al: Long noncoding RNA as modular scaffold of histone modification complexes. Science 329: 689-693, 2010.

33. Wu ZH, Wang XL, Tang HM, et al: Long non-coding RNA HOTAIR is a powerful predictor of metastasis and poor prognosis and is associated with epithelial-mesenchymal transition in colon cancer. Oncol Rep 32: 395-402, 2014.

34. Sørensen KP, Thomassen M, Tan Q, et al: Long non-coding RNA HOTAIR is an independent prognostic marker of metastasis in estrogen receptor-positive primary breast cancer. Breast Cancer Res Treat 142: 529-536, 2013.

35. Qiu JJ, Lin YY, Ye LC, et al: Overexpression of long non-coding RNA HOTAIR predicts poor patient prognosis and promotes tumor metastasis in epithelial ovarian cancer. Gynecol Oncol 134: 121-128, 2014.

36. Niinuma T, Suzuki H, Nojima M, et al: Upregulation of miR-196a and HOTAIR drive malignant character in gastrointestinal stromal tumors. Cancer Res 72: 1126-1136, 2012.

37. Liu XH, Liu ZL, Sun M, Liu J, Wang ZX and De W: The long non-coding RNA HOTAIR indicates a poor prognosis and promotes metastasis in non-small cell lung cancer. BMC Cancer 13: 464, 2013.

38. Kogo R, Shimamura T, Mimori K, et al: Long noncoding RNA HOTAIR regulates polycomb-dependent chromatin modification and is associated with poor prognosis in colorectal cancers. Cancer Res 71: 6320-6326, 2011.

39. Kim K, Jutooru I, Chadalapaka G, et al: HOTAIR is a negative prognostic factor and exhibits pro-oncogenic activity in pancreatic cancer. Oncogene 32: 1616-1625, 2013.

40. Geng YJ, Xie SL, Li Q, Ma J and Wang GY: Large intervening non-coding RNA HOTAIR is associated with hepatocellular carcinoma progression. J Int Med Res 39: 2119-2128, 2011.

41. Ding C, Cheng S, Yang Z, et al: Long non-coding RNA HOTAIR promotes cell migration and invasion via down-regulation of RNA binding motif protein 38 in hepatocellular carcinoma cells. Int J Mol Sci 15: 4060-4076, 2014.

42. Cui L, Xie XY, Wang H, Chen XL, Liu SL and Hu LN: Expression of long non-coding RNA HOTAIR mRNA in ovarian cancer. Sichuan Da Xue Xue Bao Yi Xue Ban 44: 57-59, 2013 (In Chinese).

43. Gupta RA, Shah N, Wang KC, et al: Long non-coding RNA HOTAIR reprograms chromatin state to promote cancer metastasis. Nature 464: 1071-1076, 2010. 
44. Tang L, Zhang W, Su B and Yu B: Long noncoding RNA HOTAIR is associated with motility, invasion, and metastatic potential of metastatic melanoma. Biomed Res Int 2013: 251098, 2013.

45. Watanabe $\mathrm{H}$ : Extracellular matrix - regulation of cancer invasion and metastasis. Gan To Kagaku Ryoho 37: 2058-2061, 2010 (In Japanese).

46. Labrie $M$ and St-Pierre Y: Epigenetic regulation of mmp-9 gene expression. Cell Mol Life Sci 70: 3109-3124, 2013.

47. Frank A, David V, Aurelie TR, Florent G, William H and Philippe B: Regulation of MMPs during melanoma progression: from genetic to epigenetic. Anticancer Agents Med Chem 12: 773-782, 2012.

48. Khaitan D, Dinger ME, Mazar J, et al: The melanoma-upregulated long noncoding RNA SPRY4-IT1 modulates apoptosis and invasion. Cancer Res 71: 3852-3862, 2011.

49. Reuter JS and Mathews DH: RNAstructure: software for RNA secondary structure prediction and analysis. BMC Bioinformatics 11: 129, 2010.

50. Hofacker IL: RNA secondary structure analysis using the Vienna RNA package. Curr Protoc Bioinformatics Chapter 12 Unit 12.2, 2004.

51. Rigoutsos I, Huynh T, Miranda K, Tsirigos A, McHardy A and Platt D: Short blocks from the noncoding parts of the human genome have instances within nearly all known genes and relate to biological processes. Proc Natl Acad Sci USA 103 6605-6610, 2006.

52. Zou Y, Jiang Z, Yu X, et al: Upregulation of long noncoding RNA SPRY4-IT1 modulates proliferation, migration, apoptosis, and network formation in trophoblast cells HTR-8SV/neo. PLoS One 8: e79598, 2013.

53. Xie HW, Wu QQ, Zhu B, et al: Long noncoding RNA SPRY4-IT1 is upregulated in esophageal squamous cell carcinoma and associated with poor prognosis. Tumour Biol: May 9, 2014 (Epub ahead of print)

54. Guil S and Esteller M: Cis-acting noncoding RNAs: friends and foes. Nat Struct Mol Biol 19: 1068-1075, 2012

55. Tennis MA, Van Scoyk MM, Freeman SV, Vandervest KM, Nemenoff RA and Winn RA: Sprouty-4 inhibits transformed cell growth, migration and invasion, and epithelial-mesenchymal transition, and is regulated by Wnt7A through PPARgamma in non-small cell lung cancer. Mol Cancer Res 8: 833-843, 2010.

56. Leeksma OC, Van Achterberg TA, Tsumura Y, et al: Human sprouty 4 , a new ras antagonist on $5 \mathrm{q} 31$, interacts with the dual specificity kinase TESK1. Eur J Biochem 269: 2546-2556, 2002.

57. Russo A, Ficili B, Candido S, et al: Emerging targeted therapies for melanoma treatment (review). Int J Oncol 45: 516-524, 2014

58. Wu CF, Tan GH, Ma CC and Li L: The non-coding RNA llme23 drives the malignant property of human melanoma cells. J Genet Genomics 40: 179-188, 2013.
59. Patton JG, Porro EB, Galceran J, Tempst $P$ and Nadal-Ginard B: Cloning and characterization of PSF, a novel pre-mRNA splicing factor. Genes Dev 7: 393-406, 1993.

60. Song X, Sun Y and Garen A: Roles of PSF protein and VL30 RNA in reversible gene regulation. Proc Natl Acad Sci USA 102: 12189-12193, 2005.

61. Wang G, Cui Y, Zhang G, Garen A and Song X: Regulation of proto-oncogene transcription, cell proliferation, and tumorigenesis in mice by PSF protein and a VL30 noncoding RNA. Proc Natl Acad Sci USA 106: 16794-16798, 2009.

62. Song X, Wang B, Bromberg M, Hu Z, Konigsberg W and Garen A: Retroviral-mediated transmission of a mouse VL30 RNA to human melanoma cells promotes metastasis in an immunodeficient mouse model. Proc Natl Acad Sci USA 99: 6269-6273, 2002.

63. Tsukahara T, Matsuda Y and Haniu H: PSF knockdown enhances apoptosis via downregulation of LC3B in human colon cancer cells. Biomed Res Int 2013: 204973, 2013.

64. Ren S, She M, Li M, et al: The RNA/DNA-binding protein PSF relocates to cell membrane and contributes cells' sensitivity to antitumor drug, doxorubicin. Cytometry A 85: 231-241, 2014.

65. Davies H, Bignell GR, Cox C, et al: Mutations of the BRAF gene in human cancer. Nature 417: 949-954, 2002.

66. Flaherty KT and McArthur G: BRAF, a target in melanoma: implications for solid tumor drug development. Cancer 116 : 4902-4913, 2010

67. Bamford S, Dawson E, Forbes S, et al: The COSMIC (Catalogue of Somatic Mutations in Cancer) database and website. Br J Cancer 91: 355-358, 2004

68. Jarkowski A 3rd and Khushalani NI: BRAF and beyond: tailoring strategies for the individual melanoma patient. J Carcinog 13: $1,2014$.

69. Flockhart RJ, Webster DE, Qu K, et al: BRAFV600E remodels the melanocyte transcriptome and induces BANCR to regulate melanoma cell migration. Genome Res 22: 1006-1014, 2012.

70. Berger MF, Levin JZ, Vijayendran K, et al: Integrative analysis of the melanoma transcriptome. Genome Res 20: 413-427, 2010.

71. Kong L, Zhang Y, Ye ZQ, et al: CPC: assess the protein-coding potential of transcripts using sequence features and support vector machine. Nucleic Acids Res 35: W345-W349, 2007.

72. Kawada K, Sonoshita M, Sakashita H, et al: Pivotal role of CXCR3 in melanoma cell metastasis to lymph nodes. Cancer Res 64: 4010-4017, 2004

73. Fu Y, Dominissini D, Rechavi G and He C: Gene expression regulation mediated through reversible $\mathrm{m}^{6} \mathrm{~A}$ RNA methylation. Nat Rev Genet 15: 293-306, 2014. 